\title{
Analysis of sum spectrum efficiency in multiple antennas multi-user MIMO cellular system for millimeter wave communications
}

\author{
JAIPREET KAUR*(D, MANINDER LAL SINGH and RAJDEEP SINGH SOHAL \\ Department of Electronics Technology, Guru Nanak Dev University, Amritsar, India \\ e-mail: jaipreetkr@yahoo.com
}

MS received 12 February 2019; revised 6 April 2019; accepted 4 June 2019

\begin{abstract}
In this paper, sum spectrum efficiency of two cell multiple-input multiple-output (MIMO) system having multiple antennas at base station (BS) and multiple user equipments (UEs) is analysed. The maximum ratio (MR) and multi-cell minimum mean squared error (M-MMSE) techniques are utilized for detection/ precoding in the uplink/downlink scenario. In this paper, two boundary cases of propagation channel model: line of sight $(\mathrm{LoS})$ and non-line of sight $(\mathrm{NLoS})$, and generalized propagation channel model of millimeter wave (mmWave) are compared using MR and MMSE detection techniques. The closed bound expressions of sum spectrum efficiency of MR are expressed and simulated, whereas for M-MMSE, Monte-Carlo simulations are performed on the basis of its weighting vector. The fundamental limits on the system performance, using large antenna arrays and many UEs satisfying practical constraints like desired signal to noise ratio (SNR), low complexity in signal processing, gain of inter cell interference and finite-dimensional channels are analysed.
\end{abstract}

Keywords. Multiple antennas multi-user MIMO; sum spectrum efficiency; maximum ratio (MR); multi-cell minimum mean squared error (M-MMSE); line of sight (LoS); non-line of sight (NLoS); millimeter wave (mmWave).

\section{Introduction}

There is an exponential increase in the demand of data capacity requirements in future generation mobile communications. To fulfil this demand within same bandwidth resources, the number of antennas at base station (BS) needs to be increased to serve multiple user equipments (UEs) simultaneously. Thus, multiple antennas multi-user multiple-input multiple-output (MIMO) system becomes a leverage for the future generation communication systems [1].

The MIMO system with a small number of antennas at BS is already functional, but these limited number of antennas does not improve the performance of system significantly. The sum spectrum efficiency can be enhanced remarkably by rising the number of antennas at BS [2]. Multiple antennas at BS can receive more energy from UEs and are capable of differentiating spatial directed signals by spatial processing techniques [3]. Equipping multiple antennas at BS is more convenient than at UEs, as UEs are end user products with compact size and operate at limited power batteries. Each additional antenna at BS also requires more transmit power and directivity, this leads to computational complexity.

Aaron Wyner developed a simple and tractable model to study the fundamental characteristics of cellular system

*For correspondence

Published online: 16 July 2019 using two cell network [4]. This model is easy to implement and controllable to a large extent. It can be modelled for realistic purposes, when multiple cells and distinct channel gains between every UE and BS are taken into consideration [5]. Marzetta [1] presented a concept for having unlimited number of antennas at BS using maximum ratio (MR) detection. The various opportunities and challenges with very large antennas at BS have been discussed in [2]. The potentials and limitations of massive MIMO have been considered in [3], making this an open research area for researchers. The practical set-up of multi-cell multiple antenna multi-user MIMO in $[6,7]$ has been realised. The energy and spectrum efficiency of the same is evaluated for both single-cell and multi-cell in $[8,9]$. In one of the works [10], the multicell multiuser massive MIMO networks with Rician flat fading has been discussed.

In the present work, the impact to sum spectrum efficiency is observed by rising number of antennas at BS, number of UEs, gain of inter cell interference and desired signal to noise ratio (SNR). The two different detection techniques i.e., maximum ratio (MR) and multi-cell minimum mean squared error (M-MMSE) have been used to analyse the system. The propagation channel models for line of sight (LoS), non-line of sight (NLoS) and millimeter wave (mmWave) are realised in this paper. The rest of paper is structured as follows: The system model is described in section 2, covering LoS, NLoS and mmWave 
channel models, and used detection techniques. Section 3 covers the formulation of sum spectrum efficiency using MR detection in LoS and NLoS for both uplink and downlink scenarios. Section 4 discusses the results of simulation and conclusion is drawn in the last section.

\section{System model}

A two-cell network based on Wyner model [4] has been considered in the present work, as shown in figure 1. Each cell has a single BS equipped with $N$ antennas and $M$ single antenna UEs traversing within that cell, such that $N \gg M$. In the uplink scenario, UEs communicate to their serving BS, while their signal might interfere to the other cell's BS. The desired channel response between $m$ th UE in cell 1 and BS in cell 1 is denoted by $\boldsymbol{g}_{1 m}^{1}$ for $m=1, \ldots, M$, and the interfering channel response between $i$ th UE in cell 2 and BS in cell 1 is denoted by $\boldsymbol{g}_{2 i}^{1}$ for $i=1, \ldots, M$. Because of reciprocity in TDD (time division duplexing), the channel is same for uplink and downlink and the channel from opposite direction in downlink is represented by its Hermitian. In the downlink scenario, the serving BS communicates to their active UEs, using linear transmit precoding. Similar to uplink, the downlink desired channel response between BS in cell 1 and its $m$ th desired UE in cell 1 is represented by $\left(\boldsymbol{g}_{1 m}^{1}\right)^{H}$ for $m=1, \ldots, M$, and the interfering channel response between BS in cell 2 and $m$ th UE in cell 1 is represented by $\left(\boldsymbol{g}_{1 m}^{2}\right)^{H}$ for $m=1, \ldots, M$. The superscript signifies the BS's cell index and subscript indicates the UE's cell index. The similar subscript and superscript denote the desired signal and the dissimilar subscript and superscript denote the interfering signal.

The ratio of inter cell and intra cell channel gain $\alpha$ account the macroscopic large-scale channel fading of the channel and is given by

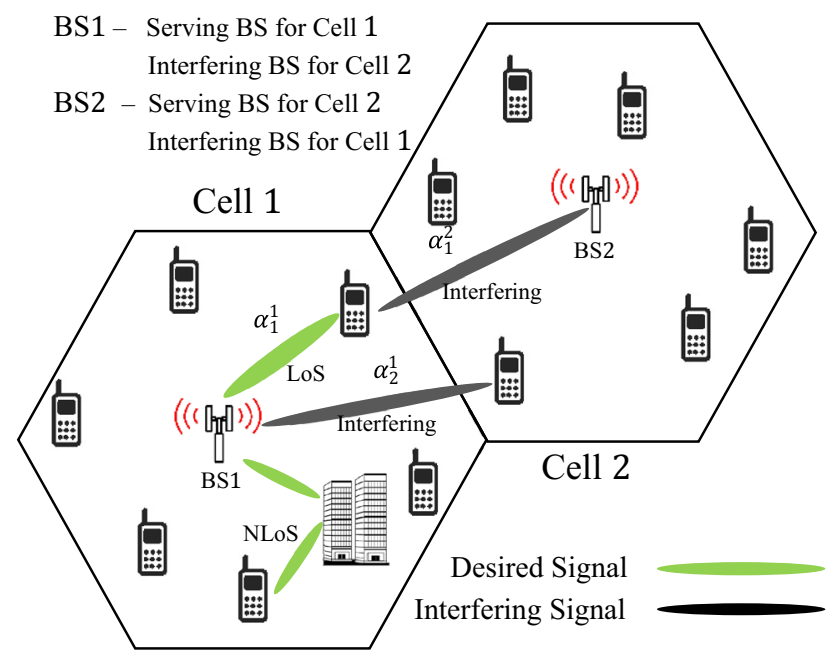

Figure 1. System model of multiple antennas multi-user MIMO two cell network.

$$
\alpha=\frac{\alpha_{2}^{1}}{\alpha_{1}^{1}}=\frac{\alpha_{1}^{2}}{\alpha_{1}^{1}}=\frac{\alpha_{2}^{1}}{\alpha_{2}^{2}}=\frac{\alpha_{1}^{2}}{\alpha_{2}^{2}} \mid 0 \leq \alpha \leq 1
$$

where $\alpha_{1}^{1}, \alpha_{2}^{2}$ are the intra cell channel gains between each UE and serving BS, which are assumed equal to each other, i.e., $\left(\alpha_{1}^{1}=\alpha_{2}^{2}\right)$, and $\alpha_{1}^{2}, \alpha_{2}^{1}$ are the inter cell channel gains between each UE and interfering BS, which are also assumed identical to each other, i.e., $\left(\alpha_{1}^{2}=\alpha_{2}^{1}\right)$. The $\alpha=1$ corresponds to cell edge case, where desired and interfering signals are equally strong.

The received uplink signal $\boldsymbol{r}_{1}$ at BS in cell 1 is modelled as follows

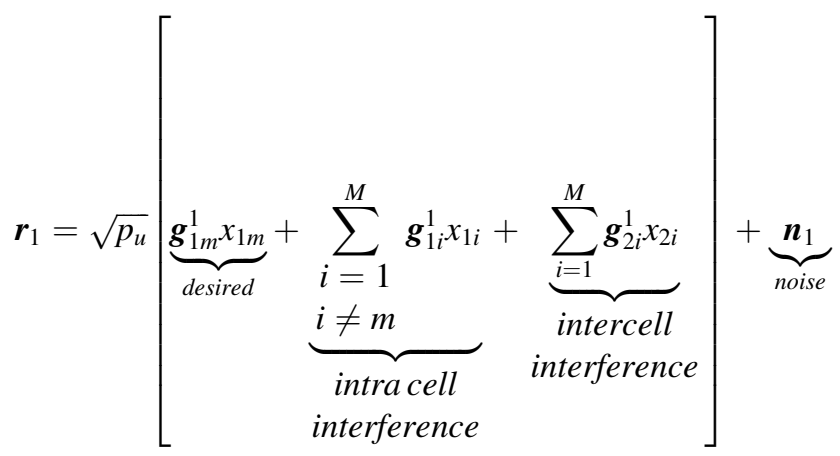

where $x_{1 m}$ is desired transmitted information signal by $m$ th UE in cell $1, x_{1 i}(i \neq m)$ and $x_{2 i}$ represent transmitted information signals by interfering UEs from cell 1 and cell 2 , respectively, $p_{u}$ is uplink transmitted power and $\boldsymbol{n}_{1} \sim \mathcal{N}_{\mathbb{C}}\left(0_{N}, \sigma^{2} \boldsymbol{I}_{N}\right)$ denotes additive white Gaussian noise (AWGN) vector in cell 1 .

The received downlink signal $r_{1 m}$ at $m$ th UE in cell 1 is given by

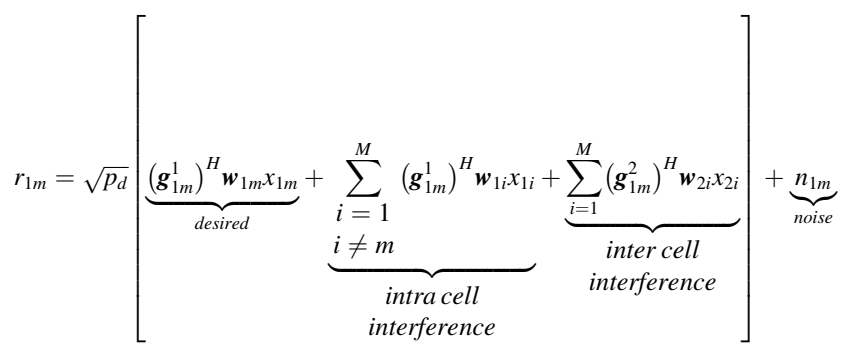

where $x_{j m}$ is the signal transmitted to $m$ th UE in cell $j$, for $j=1,2$ and $\boldsymbol{w}_{j m}$ is the unit norm precoding vector $\left(\left\|\boldsymbol{w}_{j m}\right\|=1\right)$ for $j=1,2$ and $p_{d}$ is downlink transmission power at BS. And the receiver AWGN at $m$ th UE is $n_{1 m} \sim \mathcal{N}_{\mathbb{C}}\left(0, \sigma^{2}\right)$.

\subsection{Channel models}

2.1a Line of sight channel model: The LoS channel response is deterministic vector since far field UEs are assumed to be static. Each BS has uniform linear array of $N$ antennas with antenna spacing $d_{A} \lambda$ meters, where $d_{A}$ denotes 
the number of wavelengths between neighbouring antennas and $\lambda$ is the wavelength of signal. The LoS channel response [11] relating to channel gain for $m$ th UE of $j$ th cell with respect to $\mathrm{BS}$ in cell 1 is given by

$$
\begin{aligned}
\boldsymbol{g}_{j m}^{1} & =\sqrt{\alpha_{j}^{1}}\left[1, e^{2 \pi j d_{A} \sin \left(\phi_{j m}^{1}\right)}, \ldots, e^{2 \pi j d_{A}(\mathrm{~N}-1) \sin \left(\phi_{j m}^{1}\right)}\right]^{\prime} \text { for } \\
j & =1,2
\end{aligned}
$$

where $\phi_{j m}^{1} \in(0,2 \pi)$ is the azimuth angle to $m$ th UE in $j$ th cell, comparative to the boresight of BS array in cell 1 . The LoS channel responses gradually become orthogonal with the increase in $N$ which leads to favourable propagation without causing mutual interference.

2.1b Non-line of sight channel model: The NLoS channel response is random variable and spatially uncorrelated over the BS antenna array. It is also known as uncorrelated Rayleigh fading and is modelled by complex Gaussian distribution having circular symmetry $\mathcal{N}_{\mathbb{C}}$ with zero mean and correlation matrix of channel gains. The NLoS channel response for $m$ th $\mathrm{UE}$ of $j$ th cell with respect to BS in cell 1 is given by

$$
\boldsymbol{g}_{j m}^{1}=\mathcal{N}_{\mathbb{C}}\left(0_{N}, \alpha_{j}^{1} \boldsymbol{I}_{N}\right) \text { for } j=1,2
$$

2.1c Millimeter wave channel model: The mixing of LoS and NLoS environments comprise a practical scenario, which is analysed by studying the behaviour of mmWave channel. It is highly sensitive to blockages, causing severe penetration and signal strength loss in NLoS environments [12]. The widely used Saleh-Valenzuela channel model [13] has been adopted for mmWave communications in this work. The mmWave spatial channel vector between $m$ th UE of $j$ th cell with respect to BS in cell 1 can be represented by $\boldsymbol{g}_{j m}^{1}$ and is given by

$$
\boldsymbol{g}_{j m}^{1}=\beta_{j m}^{1(0)} \boldsymbol{a}\left(\theta_{j m}^{1(0)}\right)+\sum_{l=1}^{L} \beta_{j m}^{1(l)} \boldsymbol{a}\left(\theta_{j m}^{1(l)}\right) \quad \text { for } \quad j=1,2
$$

where $\beta_{j m}^{1(0)} \boldsymbol{a}\left(\theta_{j m}^{1(0)}\right)$ represents LoS element of $m$ th UE with complex gain denoted by $\beta_{j m}^{1(0)}$ and array's steering vector presented by $\boldsymbol{a}\left(\theta_{j m}^{1(0)}\right)$. The term $\beta_{j m}^{1(l)} \boldsymbol{a}\left(\theta_{j m}^{1(l)}\right)$ represents $l$ th NLoS element of $m$ th UE for $1 \leq l \leq L$, where $L$ is total number of NLoS elements. $\boldsymbol{a}\left(\theta^{1}\right)$ is $(N \times 1)$ array steering vector for $N$ antennas in cell 1 is given by

$$
\boldsymbol{a}\left(\theta^{1}\right)=\frac{1}{\sqrt{N}}\left[\begin{array}{c}
1 \\
e^{-j 2 \pi \theta^{1}} \\
\vdots \\
e^{-j 2 \pi(N-1) \theta^{1}}
\end{array}\right]=\frac{1}{\sqrt{N}}\left[\begin{array}{c}
e^{-\frac{j 2 \pi}{\lambda} d} d \sin \phi^{1} \\
\vdots \\
e^{-\frac{j 2 \pi}{\lambda}(N-1) d \sin \phi^{1}}
\end{array}\right]
$$

where $\theta^{1}$ is spatial angle given by $\frac{d}{\lambda} \sin \phi^{1}, \phi^{1}$ is physical direction covering one sided spatial horizon satisfying $-\frac{\pi}{2} \leq \phi^{1} \leq \frac{\pi}{2}, \lambda$ is wavelength of mmWave signal and $d$ is spacing between antennas, satisfying $d=\frac{\lambda}{2}$ at mmWave frequencies [14]. The amplitudes of NLoS components $\left\{\left|\beta_{j m}^{(l)}\right|\right\}_{l=1}^{L}$ are quite feebler than the amplitude of LoS component $\beta_{j m}^{(0)}$, making mmWave channel sparse.

\subsection{Detection/precoding techniques}

It is assumed to have a perfect knowledge of channel responses at BS for uplink and at UEs for downlink and from them, the weighting vector of detection/precoding techniques [8,9] are evaluated. The linear detection techniques like MR and multi-cell minimum mean squared error (MMMSE) have been considered over the nonlinear techniques because latter increase the computational complexity of the system. The linear detection techniques also give optimal performance over large number of antennas at BS.

For uplink, detection techniques at BS are employed to detect transmitted symbol $\hat{x}_{1 m}$ by multiplying the Hermitian of weighting detection vector $\left(\boldsymbol{w}_{1 m}^{M R}\right)^{H}$ for MR detection or $\left(\boldsymbol{w}_{1 m}^{M-M M S E}\right)^{H}$ for M-MMSE detection, with the received signal $\boldsymbol{r}_{1}$ given in (2), and are respectively given as

$$
\begin{gathered}
\hat{x}_{1 m}=\left(\boldsymbol{w}_{1 m}^{M R}\right)^{H} \boldsymbol{r}_{1} \\
=\left(\boldsymbol{w}_{1 m}^{M R}\right)^{H}\left\{\sqrt{p_{u}}\left[\boldsymbol{g}_{1 m}^{1} x_{1 m}+\sum_{i=1}^{M} \boldsymbol{g}_{1 i}^{1} x_{1 i}+\sum_{i=1}^{M} \boldsymbol{g}_{2 i}^{1} x_{2 i}\right]+\boldsymbol{n}_{1}\right\} \\
\hat{x}_{1 m}=\left(\boldsymbol{w}_{1 m}^{M-M M S E}\right)^{H} \boldsymbol{r}_{1}=\left(\boldsymbol{w}_{1 m}^{M-M M S E}\right)^{H} \\
\left.\sqrt{p_{u}}\left[\begin{array}{c}
\boldsymbol{g}_{1 m}^{1} x_{1 m}+\sum_{i=1}^{M} \boldsymbol{g}_{1 i}^{1} x_{1 i}+\sum_{i=1}^{M} \boldsymbol{g}_{2 i}^{1} x_{2 i} \\
i \neq m
\end{array}\right]+\boldsymbol{n}_{1}\right\}
\end{gathered}
$$

For downlink, precoding techniques to transmitted data are employed at BS before the transmission of signal as shown in (3), where weighting precoding vector is $\boldsymbol{w}_{j m}^{M R}$ for MR precoding or $\boldsymbol{w}_{j m}^{M-M M S E}$ for M-MMSE precoding, which is multiplied with transmitted symbol $x_{j m}$.

2.2a Maximum ratio: $\quad$ MR is relatively simpler than most of the detection/precoding techniques, whose weighting vector $\boldsymbol{w}_{1 m}^{M R}$ is given by

$$
\boldsymbol{w}_{1 m}^{M R}=\frac{\boldsymbol{g}_{1 m}^{1}}{\left\|\boldsymbol{g}_{1 m}^{1}\right\|}
$$

This weighing vector maximizes the desired signal, but does not affect the interfering components. So, it is suitable for noise limited environments. 
2.2b Multi-cell minimum mean squared error: In contrast to MR, M-MMSE detection scheme not only amplifies the desired signal, but also suppresses the interfering signals from all cells and its weighting vector $\boldsymbol{w}_{1 m}^{M-M M S E}$ [5] is given by

$\boldsymbol{w}_{1 m}^{M-M M S E}=p\left[p \sum_{i=1}^{M} \boldsymbol{g}_{1 i}^{1}\left(\boldsymbol{g}_{1 i}^{1}\right)^{H}+\sum_{i=1}^{M} \boldsymbol{g}_{2 i}^{1}\left(\boldsymbol{g}_{2 i}^{1}\right)^{H}+\sigma^{2} \boldsymbol{I}_{N}\right]^{-1} \boldsymbol{g}_{1 m}^{1}$

where $p=p_{u}$ for uplink detection and $p=p_{d}$ for downlink precoding, $\boldsymbol{I}_{N}$ is identity matrix, and $\sigma^{2}$ is the noise power. This detection technique minimizes the mean squared error (MSE) given by $\mathbb{E}\left[\left|x_{1 m}-\left(\boldsymbol{w}_{1 m}^{M-M M S E}\right)^{H} r_{1}\right|^{2}\right]$.

\section{Formulation of sum spectrum efficiency}

The sum spectrum efficiency is measured in bits/s/Hz/cell. For uplink, it is from all UEs in both desired and interfering cells to BS in a desired cell. Whereas for downlink, it is from BS of both desired and interfering cell to UEs of a desired cell. The formulation of sum spectrum efficiency for MR detection in LoS and NLoS for uplink and downlink is carried in the following section.

\subsection{Uplink sum spectrum efficiency}

The signal to interference noise ratio (SINR) of $m$ th user can be obtained for MR detection by substituting $\boldsymbol{w}_{1 m}^{M R}$ from (10) to (8) and is given by

$\operatorname{SINR}_{m}$

$$
=\frac{\frac{\left|\left(g_{1 m}^{1}\right)^{H} \boldsymbol{g}_{1 m}^{1}\right|^{2}}{\left\|\boldsymbol{g}_{1 m}\right\|^{2}} p_{u}}{\sum_{\substack{i=1 \\ i \neq m}}^{M} \frac{\left|\left(g_{1 m}^{1}\right)^{H} \boldsymbol{g}_{1 i}^{1}\right|^{2}}{\left\|\boldsymbol{g}_{1 m}^{1}\right\|^{2}} p_{u}+\sum_{i=1}^{M} \frac{\left|\left(\boldsymbol{g}_{1 m}^{1}\right)^{H} \boldsymbol{g}_{2 i}^{1}\right|^{2}}{\left\|\boldsymbol{g}_{1 m}^{1}\right\|^{2}} p_{u}+\frac{\left(\left(\boldsymbol{g}_{1 m}^{1}\right)^{H}\right)^{2}}{\left\|\boldsymbol{g}_{1 m}^{1}\right\|^{2}} \sigma^{2}}
$$

3.1a For line of sight scenarios: The uplink sum spectrum efficiency $\operatorname{Rsum}_{1 u}^{\operatorname{LoS}}[9,15]$ for desired UE in cell 1 in LoS environment using MR detection from (12) is given as

$$
\begin{aligned}
& \operatorname{Rsum}_{1 u}^{L o S}=\sum_{m=1}^{M} \log _{2} \\
& \left(\begin{array}{c}
1+\frac{\left\|\boldsymbol{g}_{1 m}^{1}\right\|^{2} p_{u}}{\sum_{i=1}^{M}} \\
\quad \frac{\left|\left(\boldsymbol{g}_{1 m}^{1}\right)^{H} \boldsymbol{g}_{1 i}^{1}\right|^{2}}{\left\|\boldsymbol{g}_{1 m}^{1}\right\|^{2}} p_{u}+\sum_{i=1}^{M} \frac{\left|\left(\boldsymbol{g}_{1 m}^{1}\right)^{H} \boldsymbol{g}_{2 i}^{1}\right|^{2}}{\left\|\boldsymbol{g}_{1 m}^{1}\right\|^{2}} p_{u}+\sigma^{2}
\end{array}\right)
\end{aligned}
$$

where $p_{u}\left\|\boldsymbol{g}_{1 m}^{1}\right\|^{2}$ is the desired signal power, $\sigma^{2}$ is the noise power and $\begin{aligned} i=1 \\ i \neq m\end{aligned}$ the interference power for $m$ th UE in cell 1 . The desired channel response $\boldsymbol{g}_{1 m}^{1}$ for $m$ th UE in cell 1 and the interfering channel response $\boldsymbol{g}_{j i}^{1}$ is given by (14) and (15), respectively.

$$
\begin{gathered}
\boldsymbol{g}_{1 m}^{1}=\sqrt{\alpha_{1}^{1}}\left[1 e^{2 \pi j d_{A} \sin \left(\phi_{1 m}^{1}\right)}, \ldots, e^{2 \pi j d_{A}(\mathrm{~N}-1) \sin \left(\phi_{1 m}^{1}\right)}\right]^{\prime} \\
\boldsymbol{g}_{j i}^{1}=\sqrt{\alpha_{j}^{1}}\left[1 e^{2 \pi j d_{A} \sin \left(\phi_{j i}^{1}\right)}, \ldots, e^{2 \pi j d_{A}(\mathrm{~N}-1) \sin \left(\phi_{j i}^{1}\right)}\right]^{\prime}
\end{gathered}
$$

For desired UE in cell 1 in $(15), j=1$, i.e. $\boldsymbol{g}_{1 i}^{1}$ represents intra cell interference with $i \neq m$ and $j=2$, i.e. $\boldsymbol{g}_{2 i}^{1}$ represents inter cell interference.

The squared magnitude of $\boldsymbol{g}_{1 m}^{1}$ is given as $\left\|\boldsymbol{g}_{1 m}^{1}\right\|^{2}=\alpha_{1}^{1} N$, and $\left(\boldsymbol{g}_{1 m}^{1}\right)^{H} \boldsymbol{g}_{j i}^{1}$ is solved in (16) and further simplified by geometric series formula in (17).

$$
\begin{gathered}
\left(\boldsymbol{g}_{1 m}^{1}\right)^{H} \boldsymbol{g}_{j i}^{1}=\sqrt{\alpha_{1}^{1} \alpha_{j}^{1}} \sum_{n=0}^{N-1}\left(e^{\left.2 \pi j d_{A}\left(\sin \left(\phi_{j i}^{1}\right)-\sin \left(\phi_{1 m}^{1}\right)\right)\right)^{n}}\right. \\
=\left\{\begin{array}{c}
\sin \left(\phi_{1 m}^{1}\right) \neq \sin \left(\phi_{j i}^{1}\right) \Rightarrow \sqrt{\alpha_{1}^{1} \alpha_{j}^{1}} \frac{\left(1-e^{2 \pi j d_{A} N\left(\sin \left(\phi_{j i}^{1}\right)-\sin \left(\phi_{1 m}^{1}\right)\right)}\right)}{\left(1-e^{2 \pi j d_{A}\left(\sin \left(\phi_{j i}^{1}\right)-\sin \left(\phi_{1 m}^{1}\right)\right)}\right)} \\
\sin \left(\phi_{1 m}^{1}\right)=\sin \left(\phi_{j i}^{1}\right) \Rightarrow \sqrt{\alpha_{1}^{1} \alpha_{j}^{1}} N
\end{array}\right\}
\end{gathered}
$$

By applying Euler's formula in a part of (17), it is further reduced to (18).

$$
\begin{aligned}
& \mid \frac{1-e^{2 \pi j d_{A} N\left(\sin \left(\phi_{j i}^{1}\right)-\sin \left(\phi_{1 m}^{1}\right)\right)}}{1-\left.e^{2 \pi j d_{A}\left(\sin \left(\phi_{j i}^{1}\right)-\sin \left(\phi_{1 m}^{1}\right)\right)}\right|^{2}} \\
& =\frac{1-\cos \left(2 \pi d_{A} N\left(\sin \left(\phi_{j i}^{1}\right)-\sin \left(\phi_{1 m}^{1}\right)\right)\right)}{1-\cos \left(2 \pi d_{A}\left(\sin \left(\phi_{j i}^{1}\right)-\sin \left(\phi_{1 m}^{1}\right)\right)\right)}
\end{aligned}
$$

The $\frac{\left|\left(\boldsymbol{g}_{1 m}^{1}\right)^{H} \boldsymbol{g}_{j i}^{1}\right|^{2}}{\left\|\boldsymbol{g}_{1 m}^{1}\right\|^{2}}$ can be solved as

$$
\begin{aligned}
& \frac{\left|\left(\boldsymbol{g}_{1 m}^{1}\right)^{H} \boldsymbol{g}_{j i}^{1}\right|^{2}}{\left\|\boldsymbol{g}_{1 m}^{1}\right\|^{2}} \\
& =\left\{\begin{array}{c}
\sin \left(\phi_{1 m}^{1}\right) \neq \sin \left(\phi_{j i}^{1}\right) \Rightarrow \frac{\alpha_{j}^{1}\left(1-\cos \left(2 \pi d_{A} N\left(\sin \left(\phi_{j i}^{1}\right)-\sin \left(\phi_{1 m}^{1}\right)\right)\right)\right)}{N\left(1-\cos \left(2 \pi d_{A}\left(\sin \left(\phi_{j i}^{1}\right)-\sin \left(\phi_{1 m}^{1}\right)\right)\right)\right)} \\
\sin \left(\phi_{1 m}^{1}\right)=\sin \left(\phi_{j i}^{1}\right) \Rightarrow \alpha_{j}^{1} N
\end{array}\right\}
\end{aligned}
$$

By substituting $\frac{\left|\left(\boldsymbol{g}_{1 m}^{1}\right)^{H} \boldsymbol{g}_{j i}^{1}\right|^{2}}{\left\|\boldsymbol{g}_{1 m}^{1}\right\|^{2}}=\alpha_{j}^{1} h\left(\phi_{1 m}^{1}, \phi_{j i}^{1}\right)$, where 


$$
\begin{aligned}
& h\left(\phi_{1 m}^{1}, \phi_{j i}^{1}\right) \\
& =\left\{\begin{array}{c}
\sin \left(\phi_{1 m}^{1}\right) \neq \sin \left(\phi_{j i}^{1}\right) \Rightarrow \frac{\left(1-\cos \left(2 \pi d_{A} N\left(\sin \left(\phi_{j i}^{1}\right)-\sin \left(\phi_{1 m}^{1}\right)\right)\right)\right)}{N\left(1-\cos \left(2 \pi d_{A}\left(\sin \left(\phi_{j i}^{1}\right)-\sin \left(\phi_{1 m}^{1}\right)\right)\right)\right)} \\
\sin \left(\phi_{1 m}^{1}\right)=\sin \left(\phi_{j i}^{1}\right) \Rightarrow N
\end{array}\right\}
\end{aligned}
$$

into (13) the sum spectrum efficiency for LoS propagation model Rsum $_{1}^{L O S}$ using MR detection is simplified and is given as

$$
\begin{aligned}
& \operatorname{Rsum}_{1 u}^{L o S}=\sum_{m=1}^{M} \log _{2} \\
& \left(1+\frac{N}{\sum_{\substack{i=1 \\
i \neq l}}^{M} h\left(\phi_{1 m}^{1}, \phi_{1 i}^{1}\right)+\alpha \sum_{i=1}^{M} h\left(\phi_{1 m}^{1}, \phi_{2 i}^{1}\right)+\frac{1}{S N R_{1}}}\right)
\end{aligned}
$$

where $S N R_{1}=\frac{p_{u}}{\sigma^{2}} \alpha_{1}^{1}$

3.1b For non-line of sight scenarios: The uplink sum spectrum efficiency $\operatorname{Rsum}_{1 u}^{N L O S}$ for desired UE in cell 1 in NLoS environment using MR detection from (12) is given as

$$
\begin{aligned}
& R_{s u m_{1 u}^{N L S}}
\end{aligned}
$$

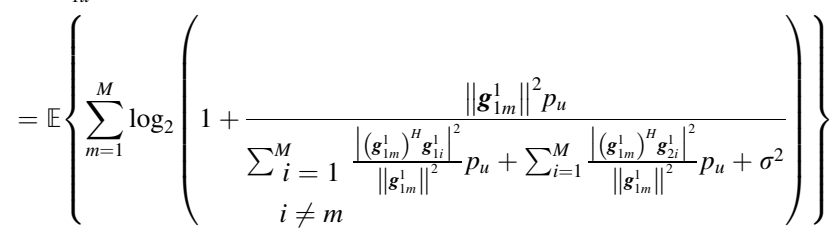

The expectation of inverse of SINR of uplink (21) can be solved as [5]

$$
\begin{aligned}
& \mathbb{E}\left\{\begin{array}{l}
\sum_{\substack{i=1 \\
i \neq m}}^{M} \frac{\left|\left(\boldsymbol{g}_{1 m}^{1}\right)^{H} \boldsymbol{g}_{1 i}^{1}\right|^{2}}{\left\|\boldsymbol{g}_{1 m}^{1}\right\|^{2}} p_{u}+\sum_{i=1}^{M} \frac{\left|\left(\boldsymbol{g}_{1 m}^{1}\right)^{H} \boldsymbol{g}_{2 i}^{1}\right|^{2}}{\left\|\boldsymbol{g}_{1 m}^{1}\right\|^{2}} p_{u}+\sigma^{2} \\
\left\|\boldsymbol{g}_{1 m}^{1}\right\|^{2} p_{u}
\end{array}\right\} \\
& =\frac{p_{u}(M-1) \alpha_{1}^{1}+p_{u} M \alpha_{2}^{1}+\sigma^{2}}{p_{u}(N-1) \alpha_{1}^{1}}
\end{aligned}
$$

where $\quad \mathbb{E}\left(\sum_{\substack{i=1 \\ i \neq m}} \frac{\left|\left(\boldsymbol{g}_{1 m}^{1}\right)^{H} \boldsymbol{g}_{1 i}^{1}\right|^{2}}{\left\|\boldsymbol{g}_{1 m}^{1}\right\|^{2}}\right)=(M-1) \alpha_{1}^{1} \quad$ an $\mathbb{E}\left(\sum_{i=1}^{M} \frac{\left|\left(\boldsymbol{g}_{1 m}^{1}\right)^{H} \boldsymbol{g}_{2 i}^{1}\right|^{2}}{\left\|\boldsymbol{g}_{1 m}^{1}\right\|^{2}}\right)=M \alpha_{2}^{1}$ because $\frac{\left|\left(\boldsymbol{g}_{1 m}^{1}\right)^{H} \boldsymbol{g}_{j i}^{1}\right|^{2}}{\left\|\boldsymbol{g}_{1 m}^{1}\right\|^{2}}$ is independent of $\boldsymbol{g}_{1 m}^{1}$ and has a mean $\alpha_{j}^{1}$ for $j=1,2$, and
$\mathbb{E}\left(\frac{1}{\left\|g_{1 m}^{1}\right\|^{2}}\right)=\frac{1}{\alpha_{1}^{1}(N-1)}$. By substituting values of (22) in (21), $\operatorname{Rsum}_{1 u}^{N L O S}$ becomes as

$$
\operatorname{Rsum}_{1 u}^{N L o S} \geq \sum_{m=1}^{M} \log _{2}\left(1+\frac{N-1}{(M-1)+M \alpha+\frac{1}{S N R_{1}}}\right)
$$

In $\operatorname{Rsum}_{1 u}^{\operatorname{LoS}}$ (20), the desired signal power strengthens linearly with $N$ and the interference term can be reduced by having well disjointed angles between UEs. Inside the logarithm of $\operatorname{Rsum}_{1 u}^{N L O S}$ (23), the desired signal gain increases with $N$ while intra cell interference gain increases by $(M-1)$ and inter cell interference gain increases linearly by $M \alpha$. With the proportional number of BS antennas relative to number of UEs, the increasing interference can be lessened.

\subsection{Downlink sum spectrum efficiency}

3.2a For line of sight scenarios: The downlink sum spectrum efficiency Rsum ${ }_{1 d}^{\operatorname{LoS}}[9,15]$ for the desired UE in cell 1 in LoS environment from (3) is given as

$$
\begin{aligned}
& \text { Rsum }_{1 d}^{\text {LoS }}= \\
& =\sum_{m=1}^{M} \log _{2}\left(1+\frac{p_{d}\left\|\boldsymbol{g}_{1 m}^{1}\right\|^{2}}{\sum_{\substack{i=1 \\
i \neq m}}^{M} p_{d} \frac{\left|\left(\boldsymbol{g}_{1 m}^{1}\right)^{H} \boldsymbol{g}_{1 i}^{1}\right|^{2}}{\left\|\boldsymbol{g}_{1 i}^{1}\right\|^{2}}+\sum_{i=1}^{M} p_{d} \frac{\left|\left(\boldsymbol{g}_{1 m}^{2}\right)^{H} \boldsymbol{g}_{2 i}^{2}\right|^{2}}{\left\|g_{2 i}^{2}\right\|^{2}}+\sigma^{2}}\right)
\end{aligned}
$$

The channel response between the BS in $c$ th cell $(c=1,2)$ and $m$ th UE in $j$ th cell is given by

$$
\left(\boldsymbol{g}_{j m}^{c}\right)^{H}=\left(\sqrt{\alpha_{j}^{c}}\left[1 e^{2 \pi j d_{A} \sin \left(\phi_{j m}^{c}\right)}, \ldots, e^{2 \pi j d_{A}(\mathrm{~N}-1) \sin \left(\phi_{j m}^{c}\right)}\right]^{\prime}\right)^{H}
$$

Equation (24) can be rewritten in (26) by using $\left\|\boldsymbol{g}_{1 m}^{1}\right\|^{2}=$ $\alpha_{1}^{1} N$ and $\frac{\left|\left(g_{1 m}^{1}\right)^{H} g_{j i}^{j}\right|^{2}}{\left\|g_{j i}^{j}\right\|^{2}}=\alpha_{1}^{j} h\left(\phi_{1 m}^{j}, \phi_{j i}^{j}\right)$ as given in (19) $\operatorname{Rsum}_{1 d}^{\text {LoS }}=\sum_{m=1}^{M} \log _{2}$

$\left(1+\frac{N}{\sum_{\substack{i=1 \\ i \neq l}}^{M} h\left(\phi_{1 m}^{1}, \phi_{1 i}^{1}\right)+\alpha \sum_{i=1}^{M} h\left(\phi_{1 m}^{2}, \phi_{2 i}^{2}\right)+\frac{1}{S N R_{1}}}\right)$ 
3.2b For non-line of sight scenarios: The downlink sum spectrum efficiency $R s u m_{1 d}^{N L S S}$ for the desired UE in cell 1 in NLoS environment using MR precoding is given as

$$
\begin{aligned}
& R \text { sum }_{1 d}^{N L O S}
\end{aligned}
$$

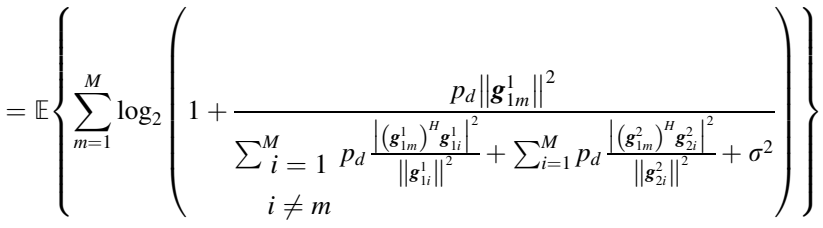

The expectation of inverse of SINR of downlink (27) can be solved as [5]

$$
\begin{aligned}
\left\{\begin{array}{l}
\sum_{i=1}^{M} p_{d} \frac{\left|\left(\boldsymbol{g}_{1 m}^{1}\right)^{H} \boldsymbol{g}_{i i}^{1}\right|^{2}}{\left\|\boldsymbol{g}_{1 i}^{1}\right\|^{2}}+\sum_{i=1}^{M} p_{d} \frac{\left|\left(\boldsymbol{g}_{1 m}^{2}\right)^{H} \boldsymbol{g}_{2 i}^{2}\right|^{2}}{\left\|g_{2 i}^{2}\right\|^{2}}+\sigma^{2} \\
p_{d}\left\|\boldsymbol{g}_{1 m}^{1}\right\|^{2}
\end{array}\right\} \\
=\sum_{i=1}^{M} \mathbb{E}\left\{\frac{\left|\left(\boldsymbol{g}_{1 m}^{1}\right)^{H} \boldsymbol{g}_{1 i}^{1}\right|^{2}}{\left\|\boldsymbol{g}_{1 i}^{1}\right\|^{2}\left\|\boldsymbol{g}_{1 m}^{1}\right\|^{2}}\right\} \\
\left.\quad+\sum_{i=1}^{M} \mathbb{E}\left\{\frac{\left|\left(\boldsymbol{g}_{1 m}^{2}\right)^{H} \boldsymbol{g}_{2 i}^{2}\right|^{2}}{\left\|\boldsymbol{g}_{2 i}^{2}\right\|^{2}}\right\}+\frac{\sigma^{2}}{p_{d}}\right) \mathbb{E}\left\{\frac{1}{\left\|\boldsymbol{g}_{1 m}^{1}\right\|^{2}}\right\} \\
=\frac{(M-1)}{N}+\frac{\left(M \alpha_{1}^{2}+\frac{\sigma^{2}}{p_{d}}\right)}{(N-1) \alpha_{1}^{1}}=\frac{(M-1) \frac{N-1}{N}+M \alpha+\frac{1}{N N R_{1}}}{N-1}
\end{aligned}
$$

where $\mathbb{E}\left\{\frac{\left|\left(g_{1 m}^{1}\right)^{H} \boldsymbol{g}_{1 i}^{1}\right|^{2}}{\left\|g_{1 i}^{1}\right\|^{2}\left\|g_{1 m}^{1}\right\|^{2}}\right\}=\frac{1}{N}, \quad \mathbb{E}\left\{\frac{\left|\left(g_{1 m}^{2}\right)^{H} \boldsymbol{g}_{2 i}^{2}\right|^{2}}{\|\left. g_{2 i}^{1}\right|^{2}}\right\}=\alpha_{1}^{2} \quad$ and $\mathbb{E}\left\{\frac{1}{\left\|g_{1 m}^{1}\right\|^{2}}\right\}=\frac{1}{(N-1) \alpha_{1}^{1}}$

By substituting values of (28) in (27), $\operatorname{Rsum}_{1 d}^{N L o S}$ becomes as

$$
\operatorname{Rsum}_{1 d}^{N L o S} \geq \sum_{m=1}^{M} \log _{2}\left(1+\frac{N-1}{(M-1) \frac{N-1}{N}+M \alpha+\frac{1}{S N R_{1}}}\right)
$$

The $\operatorname{Rsum}_{1 d}^{L O S}$ (26) is similar to $\operatorname{Rsum}_{1 u}^{\operatorname{LoS}}$ (20) with the differences of angles in the expression, and $R_{s u m}{ }_{1 d}^{N L O S}$ (29) is similar to $\operatorname{Rsum}_{1 u}^{N L O S}$ (23) with an additional product term $\frac{N-1}{N}$ in the denominator of $R_{s u m}^{N L o S}$, which becomes unity for large $N$.

\section{Simulation results}

The sum spectrum efficiency (Rsum) is characterised by the number of antennas at BS $N$, number of UEs $M$, ratio of inter cell and intra cell channel gain $\alpha$, SNR of the desired signal. Due to reciprocity of the channel and assumptions considered in Wyner model, the uplink simulations scenario is valid for downlink scenario also, so only uplink system is considered for simulations. For generalization of simulation results, the value of SNR is kept at $0 \mathrm{~dB}, \alpha=-10 \mathrm{~dB}$ and $d_{A}=1 / 2$. For MR under LoS and NLoS environments analytical results from (20) and (23) respectively are plotted, whereas for MR in mmWave and for M-MMSE in all environments, simulated results are plotted.

It is observed from figures 2 and 3 that as number of antennas at BS $N$ increases [5], Rsum increases many folds. For a smaller number of antennas at BS i.e., $N=10$, slope remains constant because limited number of spatial degrees of freedom are available at BS to accommodate UEs. At larger values of $N$, slope of Rsum becomes almost steadier and at $N=200$, it approaches linearity.

Figures 4 and 5 show plots of Rsum versus $N$, and imply that $N>40$ should be the minimum requirement of the system. The increase in $M$ from 10 to 40 shows significant rise in Rsum. Whereas beyond 40, the improvement factor does not change considerably.

These findings are observed in both MR (figure 4) and MMMSE (figure 5) detection. The performance of MR detection is better in LoS than NLoS, and NLoS than mmWave due to ignoring of interferences by MR. NLoS and mmWave have considerable interference than LoS, where in LoS only parallel signals arrive at the desired UEs which leads to minimal interference. Whereas M-MMSE behaves

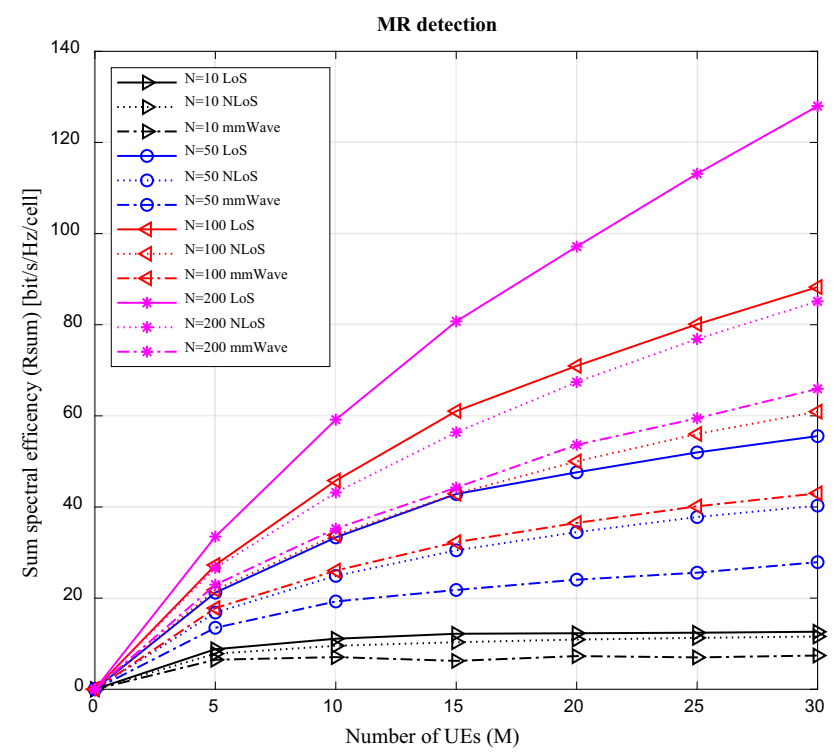

Figure 2. Sum spectrum efficiency (Rsum) v/s number of UEs $(M)$ using MR detection. 


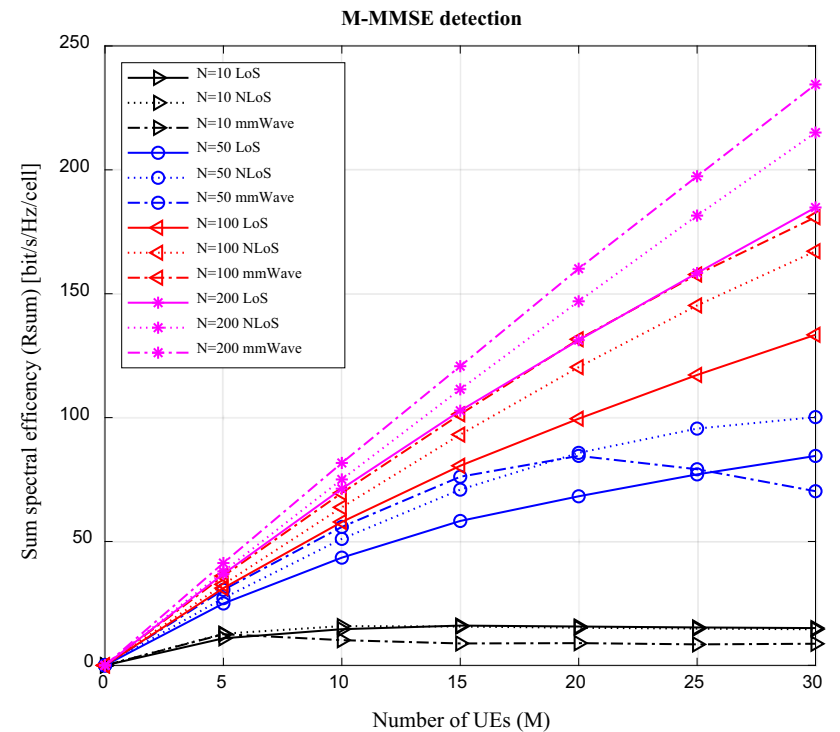

Figure 3. Sum spectrum efficiency (Rsum) v/s number of UEs (M) using M-MMSE detection.

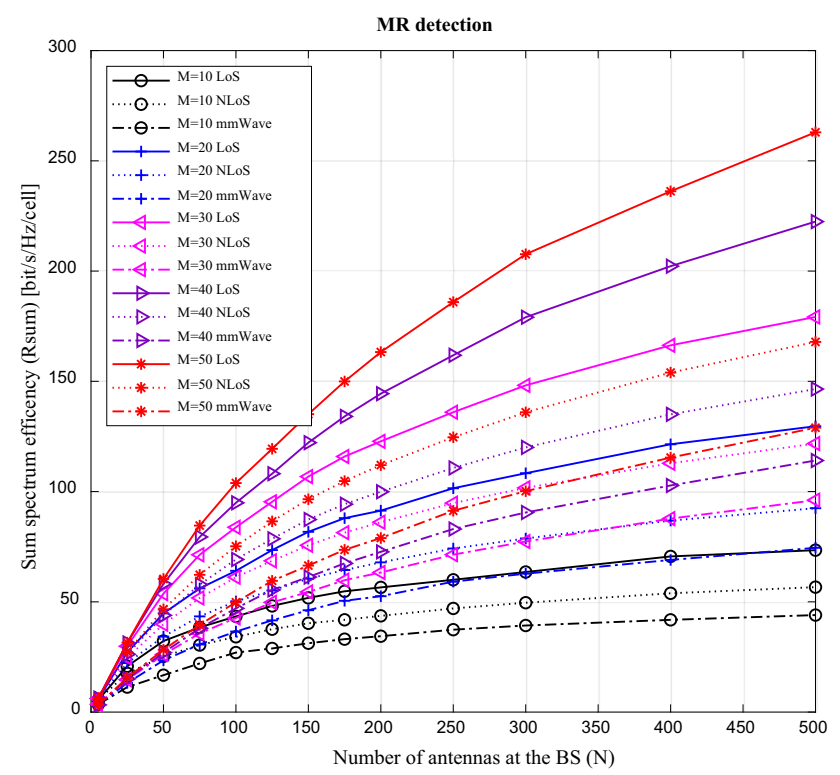

Figure 4. Sum spectrum efficiency (Rsum) v/s number of antennas at the $\mathrm{BS}(N)$ using MR detection.

opposite i.e., the mmWave system has better results than NLoS and NLoS than LoS. Because, in NLoS there are many UEs which interfere with desired UE, resulting in a large sum interference power, whereas in LoS there are limited interfering UEs having similar angles as of desired UE, causing strong interference. For MR detection, the interference is ignored so, Rsum of NLoS is lower than LoS. Whereas, M-MMSE works on interference cancellation, it is easier for M-MMSE to reject interference in NLoS than in LoS, as channels of interfering UEs may be nearly parallel to desired UE in LoS. It is even easier in mmWave to reject interference. But, overall M-MMSE detection (figure 5)

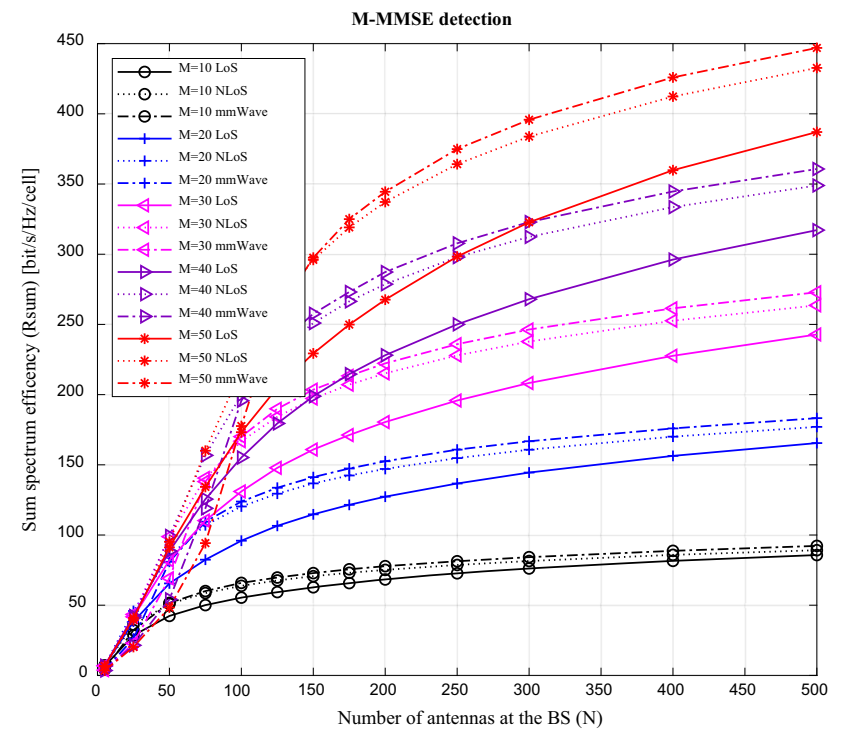

Figure 5. Sum spectrum efficiency (Rsum) v/s number of antennas at the BS $(N)$ using M-MMSE detection.

shows a far better performance as compared to MR (figure 4) in the above three environments.

The BS antenna to UE ratio is represented by $N / M \in\{1$, $2,4,8\}$ is a constant. For M-MMSE detection figures 6 and 7 show a linear increase of Rsum at increase of $N / M$ with respect to $M$ and $N$, respectively, because large gap between $N$ and $M(N \gg M)$, leads to reduction in interference. From figure 6 for mmWave at $M=14,78 \%$ increase of gain in Rsum by first fold (doubling) of $N / M$, another 53\% increase in second fold and yet another $35 \%$ in third fold are observed. Whereas, the latter folds do not

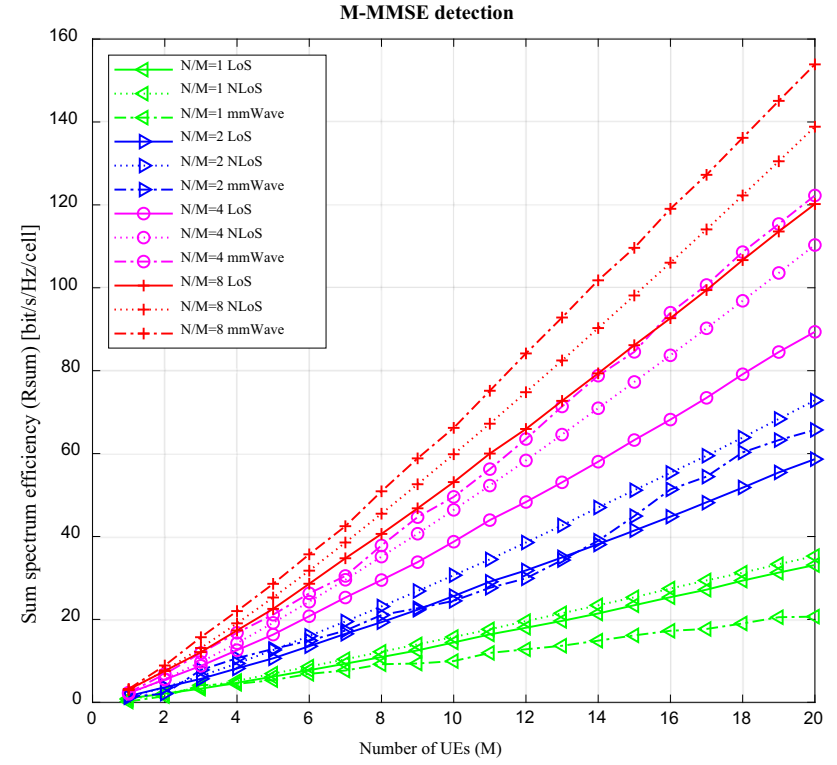

Figure 6. Sum spectrum efficiency (Rsum) v/s number of UEs $(M)$ with fixed BS antenna to UE ratio $N / M \in\{1,2,4,8\}$ using M-MMSE detection. 


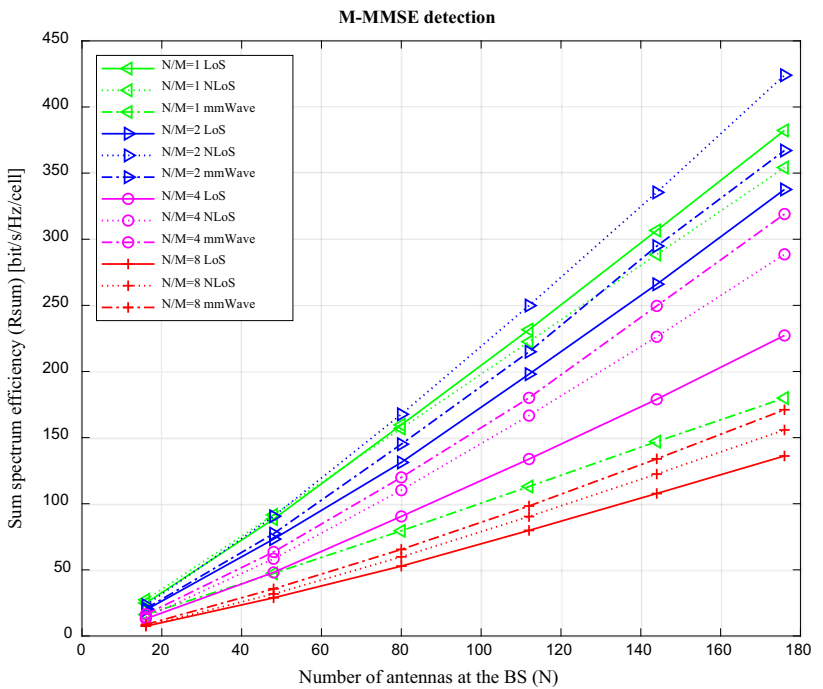

Figure 7. Sum spectrum efficiency (Rsum) v/s number of antennas at the BS $(N)$ with fixed BS antenna to UE ratio $N / M \in\{1,2,4,8\}$ using M-MMSE detection.

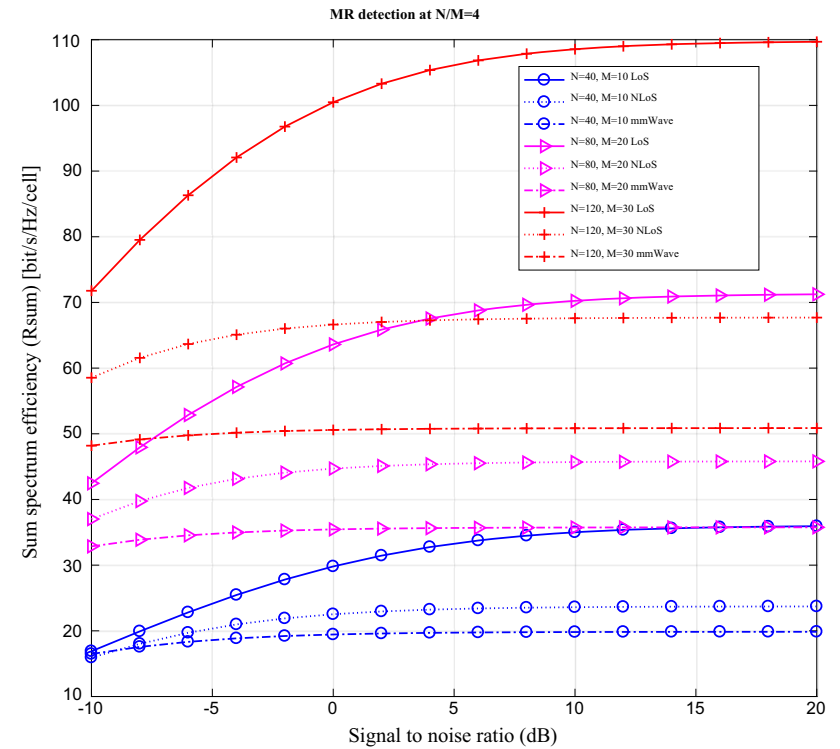

Figure 8. Sum spectrum efficiency $($ Rsum $)$ v/s signal to noise ratio keeping $\mathrm{BS}$ antenna to $\mathrm{UE}$ ratio $N / M=4$ using $\mathrm{MR}$ detection.

make significant changes, so $N / M \geq 4$ is treated as optimal ratio. Similarly, from figure 7 for mmWave, at $N=144$, $70 \%$ increase of gain in Rsum by first fold of $N / M, 46 \%$ increase in second fold and $14 \%$ in third fold for mmWave are observed. In this case too, the latter folds do not make significant changes, so $N / M \geq 4$ is treated as optimal ratio for multiple antennas multi-user MIMO system.

The performance evaluation of system using MR and MMMSE detection against SNR by keeping $N / M=4$ is shown in figures 8 and 9, respectively. In MR (figure 8), for fairly low SNR values, a finite increase in Rsum is

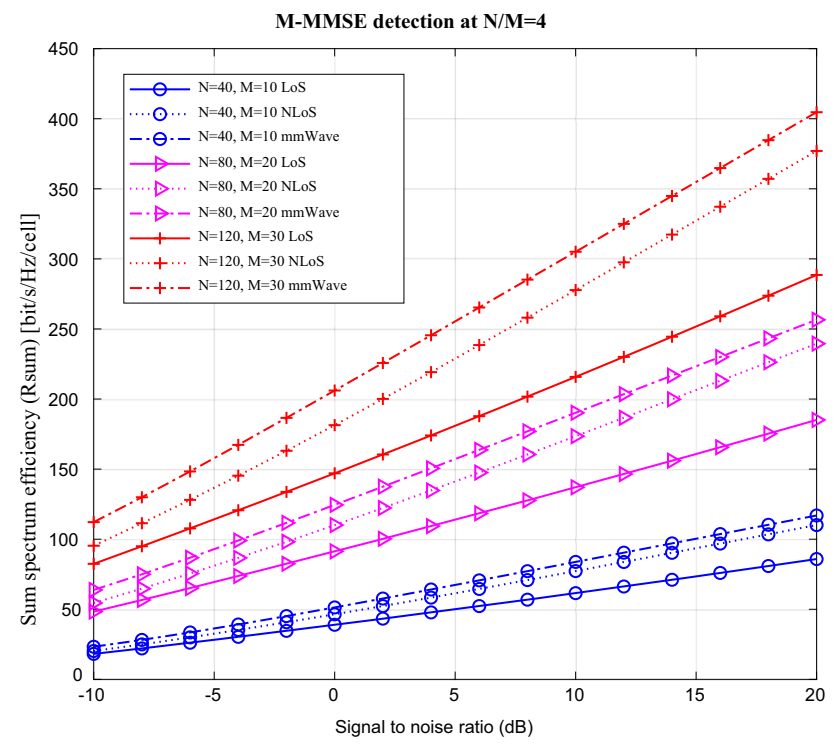

Figure 9. Sum spectrum efficiency (Rsum) v/s signal to noise ratio keeping $\mathrm{BS}$ antenna to UE ratio $N / M=4$ using M-MMSE detection.

observed. After SNR $=10 \mathrm{~dB}$ the slope becomes relatively steadier. In M-MMSE (figure 9) Rsum improves linearly with the increase of SNR value. It can be deduced from these two figures that, among them, M-MMSE plays much better role than MR.

The impact of ratio of inter cell to intra cell channel gain $\alpha$ is observed for $N=80, M=20$ by taking three different cases: $\alpha=-20 \mathrm{~dB}, \alpha=-10 \mathrm{~dB}$ and $\alpha=0 \mathrm{~dB}$ in figures 10 and 11 using MR and M-MMSE detection, respectively.

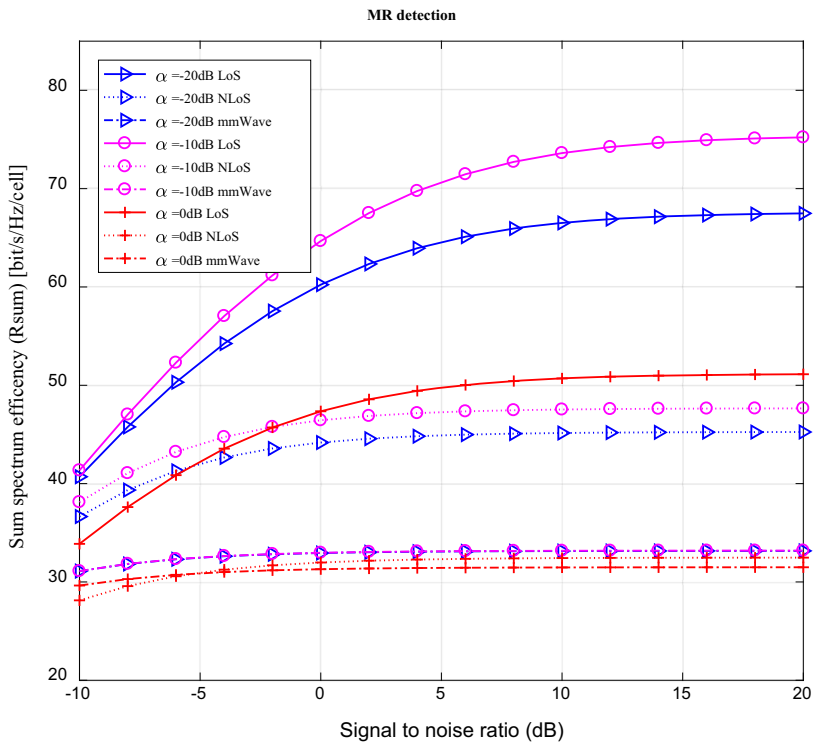

Figure 10. Sum spectrum efficiency (Rsum) v/s signal to noise ratio at $N=80, M=20$ for different values of $\alpha$ using MR detection. 


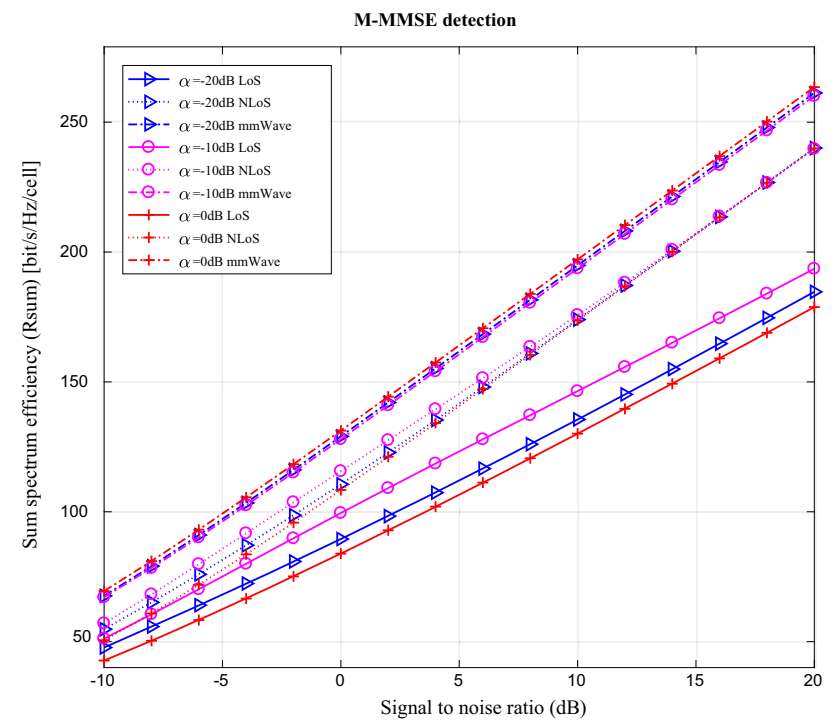

Figure 11. Sum spectrum efficiency (Rsum) v/s signal to noise ratio at $N=80, M=20$ for different values of $\alpha$ using M-MMSE detection.

There is a significant influence of $\alpha$ in MR (figure 10), whereas a little difference is observed in M-MMSE (figure 11). M-MMSE behaves better even with the lower values of $\alpha$. By fixing SNR $=20 \mathrm{~dB}$, M-MMSE yields Rsum $=177.5 \mathrm{bit} / \mathrm{s} / \mathrm{Hz} /$ cell at $\alpha=0 \mathrm{~dB}$, whereas MR gives Rsum $=78.05 \mathrm{bit} / \mathrm{s} / \mathrm{Hz} /$ cell at $\alpha=20 \mathrm{~dB}$. This can be deduced from above values that M-MMSE has better results even with lower value of $\alpha$ as compared to MR with higher values of $\alpha$.

\section{Conclusion}

By increasing the number of antennas at BS and serving multiple UEs simultaneously, sum spectrum efficiency is improved significantly. The performance improves at different rates with increase in number of antennas at BS and UEs for different propagation environments. The optimum antenna to UE ratio $N / M$ is concluded from the simulations to be 4 for mmWave. MR and M-MMSE detection upgrade sum spectrum efficiency by exploiting the array gain and latter one additionally limits the interference. For M-MMSE detection, computation becomes more complex, as the dimension of channel inverse matrix increases with increase in number of BS antennas and number of UEs. For M-MMSE, mmWave gives slightly better sum spectrum efficiency than NLoS and quite good than LoS, whereas MR shows reverse response. M-MMSE works better even for the relatively higher values of inter channel interference, while MR does not perform same even for the lower values of inter channel interference. Multiple antennas multi user MIMO cellular systems have the potential to deliver high reliability and throughputs in mmWave than in NLoS and LoS propagation environments.

\section{References}

[1] Marzetta T L 2010 Noncooperative cellular wireless with unlimited numbers of base station antennas. IEEE Trans. Wirel. Commun. 9(11): 3590-3600

[2] Rusek F, Persson D, Lau B K, Larsson E G, Marzetta T L, Edfors O and Tufvesson F 2013 Scaling up MIMO: opportunities and challenges with very large arrays. IEEE Signal Process. Mag. 30(1): 40-60

[3] Larsson E G, Edfors O, Tufvesson F E and Marzetta T L 2014 Massive MIMO for next generation wireless systems IEEE Commun. Mag. 52(2): 186-195

[4] Wyner A D 1994 Shannon-theoretic approach to Gaussian cellular multiple-access channel. IEEE Trans. Inf. Theory 40(6): 1713-1727

[5] Bjornson E, Hoydis J and Sanguinetti L 2017 Massive MIMO networks: spectral, energy, and hardware efficiency. Found. Trends 11(3-4): 154-655

[6] Shepard C, Yu H, Anand N, Li E, Marzetta T, Yang R and Zhong L 2012 Argos: practical many-antenna base stations. In: Proc. ACM MobiCom, Istanbul, Turkey. pp. 53-64

[7] Shepard C, Ding J, Guerra R E and Zhong L 2016 Understanding real many-antenna MU-MIMO channels. In: 50th Asilomar conference on signals, systems and computers, Pacific Grove, $C A$

[8] Ngo H Q, Larsson E G and Marzetta T L 2013 Energy and spectral efficiency of very large multiuser MIMO systems. IEEE Trans. Commun. 61(4): 1436-1449

[9] Cao J, Wang D, Li J and Sun Q 2017 Uplink spectral efficiency analysis of multi-cell multi-user massive MIMO over correlated Ricean channel. Sci. China Inf. Sci. 61: 1-14

[10] Kazemi M and Aghaeinia H 2016 A lower bound on achievable rate of MRT precoding in multicell multiuser massive MIMO networks with Rician flat fading. Int. J. Commun. Syst. 29(18): 2632-2649

[11] Tataria H, Smith P J, Greenstein L J, Dmochowski P A and Matthaiou M 2017 Impact of line-of-sight and unequal spatial correlation on uplink MU-MIMO systems. IEEE Wirel. Commun. Lett. 6(5): 634-637

[12] Bai T and Heath R W 2015 Coverage and rate analysis for millimeter-wave cellular networks. IEEE Trans. Wirel. Commun. 14(2): 1100-1114

[13] Sayeed A and Brady J 2013 Beamspace MIMO for highdimensional multiuser communication at millimeter-wave frequencies. In: GLOBECOM - IEEE Global Telecommunication Conference, pp. 3679-3684

[14] Brady J, Behdad N and Sayeed A M 2013 Beamspace MIMO for millimeter-wave communications: System architecture, modeling, analysis, and measurements. IEEE Trans. Antennas Propag. 61(7): 3814-3827

[15] Ngo H Q 2012 Performance Bounds for Very Large Multiuser MIMO Systems. Licentiate dissertation, Linköping 